\title{
NUMERICAL QUENCHING FOR HEAT EQUATIONS WITH COUPLED NONLINEAR BOUNDARY FLUX
}

\section{KOUAMÉ BÉRANGER EDJA ${ }^{1, *}$, KOFFI N'GUESSAN ${ }^{2}$, BROU JEAN-CLAUDE KOUA $^{3}$ AND KIDJEGBO AUGUSTIN TOURÉ ${ }^{1}$}

\author{
${ }^{1}$ Institut National Polytechnique Houphouët-Boigny Yamoussoukro, BP 2444, Côte d'Ivoire \\ ${ }^{2}$ UFR SED, Université Alassane Ouattara, 01 BP V 18 Bouaké 01, Côte d'Ivoire \\ ${ }^{3}$ UFR Mathématique et Informatique, Université Félix Houphouët Boigny, Côte d'Ivoire
}

${ }^{*}$ Corresponding author: kouame.edja@inphb.ci

\begin{abstract}
In this paper, we study a numerical approximation of the following problem $u_{t}=u_{x x}, v_{t}=v_{x x}$, $0<x<1,0<t<T ; u_{x}(0, t)=u^{-m}(0, t)+v^{-p}(0, t), v_{x}(0, t)=u^{-q}(0, t)+v^{-n}(0, t)$ and $u_{x}(1, t)=$ $v_{x}(1, t)=0,0<t<T$, where $m, p, q$ and $n$ are parameters. We prove that the solution of a semidiscrete form of above problem quenches in a finite time only at first node of the mesh. We show that the time derivative of the solution blows up at quenching node. Some conditions under which the non-simultaneous or simultaneous quenching occurs for the solution of the semidiscrete problem are obtained. We establish the convergence of the quenching time. Finally, some numerical results to illustrate our analysis are given.
\end{abstract}

\section{INTRODUCTION}

In this paper, we study the behavior of a semidiscrete approximation of the following heat equations involving nonlinear boundary flux conditions :

$$
u_{t}(x, t)=u_{x x}(x, t), \quad v_{t}(x, t)=v_{x x}(x, t),(x, t) \in(0,1) \times(0, T),
$$

Received 2019-08-08; accepted 2019-09-23; published 2019-11-01.

2010 Mathematics Subject Classification. 65M06, 65M12, 35K05, 35K55.

Key words and phrases. Numerical quenching; non-simultaneous; heat equation; nonlinear boundary.

(C)2019 Authors retain the copyrights of their papers, and all open access articles are distributed under the terms of the Creative Commons Attribution License. 


$$
\begin{aligned}
& u_{x}(0, t)=u^{-m}(0, t)+v^{-p}(0, t), v_{x}(0, t)=u^{-q}(0, t)+v^{-n}(0, t), t \in(0, T), \\
& u_{x}(1, t)=0, \quad v_{x}(1, t)=0, \quad t \in(0, T), \\
& u(x, 0)=u_{0}(x), \quad v(x, 0)=v_{0}(x), \quad x \in[0,1],
\end{aligned}
$$

where $m, n \geq 0, p, q>0, u_{0}$ and $v_{0}$ are positive smooth functions satisfying the compatibility conditions $u_{0}^{\prime}(0)=u_{0}^{-m}(0)+v_{0}^{-p}(0), v_{0}^{\prime}(0)=u_{0}^{-q}(0)+v_{0}^{-n}(0), u_{0}^{\prime}(1)=0, v_{0}^{\prime}(1)=0$, and $u_{0}^{\prime}, v_{0}^{\prime} \geq 0$ and $u_{0}^{\prime \prime}, v_{0}^{\prime \prime}<0$ on $(0,1]$.

Here $[0, T)$ is the maximal time interval such that

$$
\forall t \in[0, T), \inf \min _{0 \leq x \leq 1}\{u(x, t), v(x, t)\}>0 .
$$

We have

$$
\lim _{t \rightarrow T^{-}} \inf \min _{0 \leq x \leq 1}\{u(x, t), v(x, t)\}=0^{+} .
$$

The time $T$ can be finite or infinite. If $T$ is finite, then we say that the solution $(u, v)$ quenches in a finite time and $T$ is called the quenching time of $(u, v)$. If $T$ is infinite, then we affirm that the solution $(u, v)$ quenches globally.

Nonlinear parabolic systems like (1.1)-(1.4) come from chemical reactions, heat transfer, etc, where $u$ and $v$ represent the temperatures of two different materials during heat propagation. The quenching phenomenon of parabolic problems has been the issue of intensive study (see for example $[3,4,8-10]$ and the references cited therein), particulary the study of heat equations system with nonlinear boundary conditions has been the subject of investigation of several authors in recent years (see $[6,7,14,15,17]$ and the references cited therein). In [7] the authors study this problem, they prove that the solution $(u, v)$ quenches in finite time $T$ and the quenching occurs only at the boundary $x=0$ for $0<u_{0}, v_{0} \leq 1$. They show that

- if $p<n+1$, there exist initial data such that the non-simultaneous quenching occurs ;

- if $q \leq \frac{n(m+1)}{n+1}$ and $p \geq n+1\left(p \leq \frac{m(n+1)}{m+1}\right.$ and $\left.q \geq m+1\right)$, the non-simultaneous quenching occurs for any positive initial data ;

- if $q \geq m+1, p \geq n+1$, any quenching must be simultaneous and obtain of results on non-simultaneous quenching rate.

Moreover, if quenching is simultaneous they found the quenching rate, which depends on the parameter in the flux associated to the other component of the initial data.

To the best of our knowledge, no studies have been performed on the numerical approximation of equations (1.1)-(1.4). In this paper, we investigate in the numerical study using a semidiscrete form of (1.1)-(1.4), 
especially in study of simultaneous and non-simultaneous quenching. For that, we consider a uniform mesh on the interval $[0,1]$

$$
x_{i}=(i-1) h, i=1, \ldots, I, h=1 /(I-1),
$$

$U_{h}(t)=\left(U_{1}(t), \ldots, U_{I}(t)\right)^{T}, V_{h}(t)=\left(V_{1}(t), \ldots, V_{I}(t)\right)^{T}$, where $U_{i}(t)$ and $V_{i}(t)$ are the values of the numerical approximation of $u$ and $v$ at the nodes $x_{i}$ at time $t$. We also denote $\varphi_{1, i}$ and $\varphi_{2, i}$, respectively, the values of the numerical approximation of $u_{0}$ and $v_{0}$ at the nodes $x_{i}$. By the finite difference method we obtain the following system of ODEs whose the solution is $\left(U_{h}, V_{h}\right)$ :

$$
\begin{array}{lr}
U_{i}^{\prime}(t)=\delta^{2} U_{i}(t)-b_{i}\left(U_{i}^{-m}(t)+V_{i}^{-p}(t)\right), & i=1, \ldots, I, t \in\left(0, T_{h}\right), \\
V_{i}^{\prime}(t)=\delta^{2} V_{i}(t)-b_{i}\left(U_{i}^{-q}(t)+V_{i}^{-n}(t)\right), & i=1, \ldots, I, t \in\left(0, T_{h}\right), \\
U_{i}(0)=\varphi_{1, i} \quad V_{i}(0)=\varphi_{2, i}, & i=1, \ldots, I,
\end{array}
$$

where

$$
\begin{gathered}
0<\varphi_{1, i} \leq M, \quad 0<\varphi_{2, i} \leq N, \quad i=1, \ldots, I \\
\delta^{2} U_{i}(t)=\frac{U_{i-1}(t)-2 U_{i}(t)+U_{i+1}(t)}{h^{2}}, 2 \leq i \leq I-1, t \in\left(0, T_{h}\right), \\
\delta^{2} U_{1}(t)=\frac{2 U_{2}(t)-2 U_{1}(t)}{h^{2}}, \quad \delta^{2} U_{I}(t)=\frac{2 U_{I-1}(t)-2 U_{I}(t)}{h^{2}}, t \in\left(0, T_{h}\right), \\
b_{1}=\frac{2}{h}, \text { and } b_{i}=0, \quad i=2, \ldots, I .
\end{gathered}
$$

Here $\left[0, T_{h}\right)$ is the maximal time interval such that

$$
\forall t \in\left[0, T_{h}\right), \inf \min _{1 \leq i \leq I}\left\{U_{i}(t), V_{i}(t)\right\}>0 .
$$

We have

$$
\lim _{t \rightarrow T_{h}^{-}} \inf \min _{1 \leq i \leq I}\left\{U_{i}(t), V_{i}(t)\right\}=0^{+} .
$$

The time $T_{h}$ can be finite or infinite. If $T_{h}$ is finite, then we say that the solution $\left(U_{h}, V_{h}\right)$ quenches in a finite time and $T_{h}$ is called the semidiscrete quenching time of $\left(U_{h}, V_{h}\right)$. If $T_{h}$ is infinite, then we affirm that the solution $\left(U_{h}, V_{h}\right)$ quenches globally.

We show that our semidiscrete scheme reproduces well the conditions for the quenching, quenching set or simultaneous and non-simultaneous quenching of system (1.1)-(1.4). By following, it is also proved that when quenching occurs, the semidiscrete quenching time converges to the theoretical one when the mesh size goes to zero and we give a result on numerical non-simultaneous quenching rate. For previous work on numerical approximations of heat equations with non-linear boundary conditions we refer to $[1,2,5,11-13,16]$ and the references cited therein. The rest of the paper is organized as follows : in the next section, we give 
some properties concerning our semidiscrete scheme. In Section 3, under some conditions, we prove that the solution of the semidiscrete scheme (1.5)-(1.7) quenches in a finite time, we give a result on numerical quenching set. We also show that the time derivative of the solution blows up at quenching node. In Section 4 a criterion to identify simultaneous and non-simultaneous quenching is proposed. In Section 5, we show the convergence of the semidiscrete scheme and the convergence of the quenching times to the theoretical one when the mesh size goes to zero. Finally, in the last section, we give some numerical results to illustrate our analysis.

\section{Properties of the SEMidiscrete scheme}

In this section, we give some auxiliary results for the problem (1.5)-(1.7).

Definition 2.1. We say that $\left(\underline{U_{h}}, \underline{V_{h}}\right) \in\left(C^{1}\left(\left[0, T_{h}\right), \mathbf{R}^{I}\right)\right)^{2}$ is a lower solution of (1.5)-(1.7) if

$$
\begin{array}{rr}
\underline{U_{i}^{\prime}}(t) \leq \delta^{2} \underline{U_{i}}(t)-b_{i}\left(\underline{U}_{i}^{-m}(t)+\underline{V}_{i}^{-p}(t)\right), & i=1, \ldots, I, t \in\left(0, T_{h}\right), \\
\underline{V_{i}^{\prime}}(t) \leq \delta^{2} \underline{V_{i}}(t)-b_{i}\left(\underline{U}_{i}^{-q}(t)+\underline{V}^{-n}(t)\right), & i=1, \ldots, I, t \in\left(0, T_{h}\right), \\
0<\underline{U_{i}}(0) \leq \varphi_{1, i}, 0<\underline{V_{i}}(0) \leq \varphi_{2, i}, & i=1, \ldots, I,
\end{array}
$$

where $\left(U_{h}, V_{h}\right)$ is the solution of (1.5)-(1.7). On the other hand, we say that

$\left(\overline{U_{h}}, \overline{V_{h}}\right) \in\left(C^{1}\left(\left[0, T_{h}\right), \mathbf{R}^{I}\right)\right)^{2}$ is an upper solution of (1.5)-(1.7) if these inequalities are reversed.

The following lemma is a discrete form of the maximum principle.

Lemma 2.1. Let $e_{h}, c_{h}, \alpha_{h}, \beta_{h} \in\left(C^{0}\left(\left[0, T_{h}\right), \mathbf{R}^{I}\right)\right.$ and $U_{h}, V_{h} \in C^{1}\left(\left[0, T_{h}\right), \mathbf{R}^{I}\right)$ such that

$$
\begin{aligned}
& U_{i}^{\prime}(t)-\delta^{2} U_{i}(t)+e_{i}(t) U_{i}(t)+c_{i}(t) V_{i}(t) \geq 0, \quad i=1 \ldots, I, t \in\left(0, T_{h}\right), \\
& V_{i}^{\prime}(t)-\delta^{2} V_{i}(t)+\alpha_{i}(t) U_{i}(t)+\beta_{i}(t) V_{i}(t) \geq 0, \quad i=1 \ldots, I, t \in\left(0, T_{h}\right), \\
& U_{i}(0) \geq 0, \quad V_{i}(0) \geq 0, \quad i=1 \ldots, I .
\end{aligned}
$$

Then we have

$$
U_{i}(t) \geq 0, V_{i}(t) \geq 0, \quad i=1 \ldots, I, t \in\left(0, T_{h}\right)
$$

Proof. Let $T_{0}<T_{h}$ and let $\left(Z_{h}(t), W_{h}(t)\right)=\left(e^{\lambda t} U_{h}(t), e^{\lambda t} V_{h}(t)\right)$ where $\lambda$ is a real. We find that $\left(Z_{h}(t), W_{h}(t)\right)$ satisfies the following inequalities :

$$
\begin{aligned}
& Z_{i}^{\prime}(t)-\delta^{2} Z_{i}(t)+\left(e_{i}(t)-\lambda\right) Z_{i}(t)+c_{i}(t) W_{i}(t) \geq 0, \quad i=1 \ldots, I, t \in\left(0, T_{h}\right), \\
& W_{i}^{\prime}(t)-\delta^{2} W_{i}(t)+\alpha_{i}(t) Z_{i}(t)+\left(\beta_{i}(t)-\lambda\right) W_{i}(t) \geq 0, i=1 \ldots, I, t \in\left(0, T_{h}\right),
\end{aligned}
$$




$$
Z_{i}(0) \geq 0, \quad W_{i}(0) \geq 0, \quad i=1 \ldots, I .
$$

Set $m=\min \left\{\min _{1 \leq i \leq I, t \in\left[0, T_{0}\right]} Z_{i}(t), \min _{1 \leq i \leq I, t \in\left[0, T_{0}\right]} W_{i}(t)\right\}$. Since for $i \in\{0, \ldots, I\}, Z_{i}(t)$ and $W_{i}(t)$ are continuous functions on a compact, we can assume that $m=Z_{i_{0}}\left(t_{i_{0}}\right)$ for a certain $i_{0} \in\{0, \ldots, I\}$.

Assume $m<0$.

Taking $\lambda$ negative such that

$$
e_{i_{0}}\left(t_{i_{0}}\right)-\lambda>0 \text { and } \beta_{i_{0}}\left(t_{i_{0}}\right)-\lambda>0 .
$$

If $t_{i_{0}}=0$, then $Z_{i_{0}}(0)<0$, which contradicts $(2.3)$, hence $t_{i_{0}} \neq 0$;

if $1 \leq i_{0} \leq I$, we have

$$
Z_{i_{0}}^{\prime}\left(t_{i_{0}}\right)=\lim _{k \rightarrow 0} \frac{Z_{i_{0}}\left(t_{i_{0}}\right)-Z_{i_{0}}\left(t_{i_{0}}-k\right)}{k} \leq 0 .
$$

Moreover by a straightforward computation we get

$$
Z_{i_{0}}^{\prime}\left(t_{i_{0}}\right)-\delta^{2} Z_{i_{0}}\left(t_{i_{0}}\right)+\left(e_{i_{0}}\left(t_{i_{0}}\right)-\lambda\right) Z_{i_{0}}\left(t_{i_{0}}\right)+c_{i_{0}}\left(t_{i_{0}}\right) W_{i_{0}}\left(t_{i_{0}}\right)<0
$$

but these inequalities contradict (2.1) and the proof is completed.

Lemma 2.2. Let $\left(\underline{U_{h}}, \underline{V_{h}}\right)$ and $\left(\overline{U_{h}}, \overline{V_{h}}\right)$ be lower and upper solutions of (1.5)-(1.7) respectively such that, $\left(\underline{U_{h}}(0), \underline{V_{h}}(0)\right) \leq\left(\overline{U_{h}}(0), \overline{V_{h}}(0)\right)$ then

$$
\left(\underline{U_{h}}(t), \underline{V_{h}}(t)\right) \leq\left(\overline{U_{h}}(t), \overline{V_{h}}(t)\right) .
$$

Proof. Let us define $\left(Z_{h}(t), W_{h}(t)\right)=\left(\overline{U_{h}}(t), \overline{V_{h}}(t)\right)-\left(\underline{U_{h}}(t), \underline{V_{h}}(t)\right)$. We obtain

$$
\begin{gathered}
Z_{i}^{\prime}(t)-\delta^{2} Z_{i}(t)-m b_{i}\left(\mu_{i}(t)\right)^{-m-1} Z_{i}(t)-p b_{i}\left(\nu_{i}(t)\right)^{-p-1} W_{i}(t) \geq 0, i=1, \ldots, I \\
W_{i}^{\prime}(t)-\delta^{2} W_{i}(t)-q b_{i}\left(\mu_{i}(t)\right)^{-q-1} Z_{i}(t)-n b_{i}\left(\nu_{i}(t)\right)^{-n-1} W_{i}(t) \geq 0, i=1, \ldots, I \\
Z_{i}(0) \geq 0, \quad W_{i}(0) \geq 0, \quad i=1, \ldots, I
\end{gathered}
$$

where $\mu_{i}(t), \nu_{i}(t)$ lie, respectively, between $\underline{U_{i}}(t)$ and $\overline{U_{i}}(t)$, and between $\underline{V_{i}}(t)$ and $\overline{V_{i}}(t)$, for $i \in\{1, \ldots, I\}$. We can rewrite (2.4)-(2.5) as

$$
\begin{gathered}
Z_{i}^{\prime}(t)-\delta^{2} Z_{i}(t)+e_{i}(t) Z_{i}(t)+c_{i}(t) W_{i}(t) \geq 0, \quad i=1, \ldots, I, t \in\left(0, T_{h}\right), \\
W_{i}^{\prime}(t)-\delta^{2} W_{i}(t)+\alpha_{i}(t) Z_{i}(t)+\beta_{i}(t) W_{i}(t) \geq 0, \quad i=1, \ldots, I, \quad t \in\left(0, T_{h}\right),
\end{gathered}
$$

where $e_{i}(t)=-m b_{i}\left(\mu_{i}(t)\right)^{-m-1}, c_{i}(t)=-p b_{i}\left(\nu_{i}(t)\right)^{-p-1}, \alpha_{i}(t)=-q b_{i}\left(\mu_{i}(t)\right)^{-q-1}$, and $\beta_{i}(t)=$ $-n b_{i}\left(\nu_{i}(t)\right)^{-n-1} i=1, \ldots, I, \forall t \in\left(0, T_{h}\right)$. According to Lemma 2.1, $Z_{i}(t) \geq 0, W i(t) \geq 0$, for $i=1, \ldots, I$, $\forall t \in\left(0, T_{h}\right)$ and the proof is completed. 
The next lemma gives the properties of the semidiscrete solution.

Lemma 2.3. Let $\left(U_{h}, V_{h}\right) \in\left(C^{1}\left(\left[0, T_{h}\right), \mathbf{R}^{I}\right)\right)^{2}$ be the solution of (1.5)-(1.7) with an initial data $\left(\varphi_{1, h}, \varphi_{2, h}\right)$ upper solution such that $0<\varphi_{1, i}<\varphi_{1, i+1} \leq M$ and $0<\varphi_{2, i}<\varphi_{2, i+1} \leq N$ for $i=1, \ldots, I-1$. Then we have

(i) $0<U_{i}(t) \leq \varphi_{1, i} \leq M$ and $0<V_{i}(t) \leq \varphi_{2, i} \leq N$, for $i=1, \ldots, I$, $t \in\left[0, T_{h}\right)$;

(ii) $\left(U_{i+1}(t), V_{i+1}(t)\right)>\left(U_{i}(t), V_{i}(t)\right), \quad i=1, \ldots, I-1, \quad t \in\left(0, T_{h}\right)$;

(iii) $\left(U_{i}^{\prime}(t), V_{i}^{\prime}(t)\right) \leq 0, \quad i=1, \ldots, I, t \in\left(0, T_{h}\right)$.

Proof. (i) Since $\left(\varphi_{1, h}, \varphi_{2, h}\right)$ is an upper solution of (1.5)-(1.7), by the Lemma 2.1 and 2.2 we have $0<U_{i}(t) \leq M$ and $0<V_{i}(t) \leq N$, for $i=1, \ldots, I, t \in\left[0, T_{h}\right)$.

(ii) We argue by contradiction. Assume, that $t_{0}$ the first $t>0$, such that $\left(K_{i}, L_{i}\right)(t)=\left(U_{i+1}-U_{i}, V_{i+1}-\right.$ $\left.V_{i}\right)(t)>0$, for $1 \leq i \leq I-1$, but $\min \left\{K_{i_{0}}\left(t_{0}\right), L_{i_{0}}\left(t_{0}\right)\right\}=0$ for a certain $i_{0} \in\{1, \ldots, I-1\}$. Assume that $K_{i_{0}}\left(t_{0}\right)=U_{i_{0}+1}\left(t_{0}\right)-U_{i_{0}}\left(t_{0}\right)=0$. Without lost of generality, we can suppose that $i_{0}$ is the smallest integer which satisfies the above equality. Therefore, by simple computation, $\left(K_{h}, L_{h}\right)$ verifies

$$
\begin{gathered}
K_{h}^{\prime}(t)=-A^{\prime} K_{h}(t)+B^{\prime} U_{h}^{-m}(t)+B^{\prime} V_{h}^{-p}(t), \\
L_{h}^{\prime}(t)=-A^{\prime} L_{h}(t)+B^{\prime} U_{h}^{-q}(t)+B^{\prime} V_{h}^{-n}(t),
\end{gathered}
$$

where

$$
A^{\prime}=\frac{1}{h^{2}}\left(\begin{array}{ccccc}
3 & -1 & 0 & \ldots & 0 \\
-1 & 2 & -1 & \ddots & \vdots \\
0 & \ddots & \ddots & \ddots & 0 \\
\vdots & \ddots & -1 & 2 & -1 \\
0 & \ldots & 0 & -1 & 3
\end{array}\right), B^{\prime}=\left(\begin{array}{ccccc}
\frac{2}{h} & 0 & 0 & \ldots & 0 \\
0 & 0 & \ddots & & \vdots \\
\vdots & \ddots & \ddots & \ddots & \vdots \\
\vdots & & \ddots & 0 & 0 \\
0 & \ldots & \ldots & 0 & 0
\end{array}\right) .
$$

On the one hand

$$
K_{i_{0}}^{\prime}\left(t_{0}\right)=\lim _{\epsilon \rightarrow 0} \frac{K_{i_{0}}\left(t_{0}\right)-K_{i_{0}}\left(t_{0}-\epsilon\right)}{\epsilon} \leq 0
$$

and, on the other hand

$$
-\sum_{j=1}^{I} a_{i_{0}, j}^{\prime} K_{j}(t)+b_{i_{0}}^{\prime} U_{i_{0}}^{m}\left(t_{0}\right)+b_{i_{0}}^{\prime} V_{i_{0}}^{p}\left(t_{0}\right)>0
$$

Thus we have a contradiction, hence we obtain the desired result. 
(iii) Denote $F_{i}(t)=U_{i}(t)-U_{i}(t+\varepsilon)$ and $G_{i}(t)=V_{i}(t)-V_{i}(t+\varepsilon)$, for $i=1, \ldots, I$, using (i) we obtain $F_{i}(0) \geq 0, G_{i}(0) \geq 0$ for $i=1, \ldots, I$. It is not hard to see that

$$
\begin{aligned}
F_{i}^{\prime}(t) & =\delta^{2} F_{i}(t)+m b_{i}\left(\xi_{i}(t)\right)^{-m-1} F_{i}(t)+p b_{i}\left(\eta_{i}(t)\right)^{-p-1} G_{i}(t) \geq 0, \\
G_{i}^{\prime}(t) & =\delta^{2} G_{i}(t)+q b_{i}\left(\xi_{i}(t)\right)^{-q-1} F_{i}(t)+n b_{i}\left(\eta_{i}(t)\right)^{-n-1} G_{i}(t) \geq 0,
\end{aligned}
$$

where $\xi_{i}(t), \eta_{i}(t)$ lie, respectively, between $U_{i}(t+\varepsilon)$ and $U_{i}(t)$ and between $V_{i}(t+\varepsilon)$ and $V_{i}(t)$. From Lemma 2.1 we get

$$
F_{i}(t) \geq 0 \text { and } G_{i}(t) \geq 0 \text { for } i=1, \ldots, I, t \in\left(0, T_{h}\right) .
$$

This fact implies the desired result.

\section{QUeNCHING AND BLOW-UP}

Let $\left(U_{h}, V_{h}\right)$ be the solution of (1.5)-(1.7) with $0<\varphi_{1, i} \leq M, 0<\varphi_{1, i} \leq N$ for $i=1, \ldots, I$. Inspired by $[4,7]$ we prove that $\left(U_{h}, V_{h}\right)$ quenches in a finite time and $\left(U_{h}^{\prime}, V_{h}^{\prime}\right)$ blows up at quenching node.

Theorem 3.1. The solution $\left(U_{h}, V_{h}\right)$ of (1.5)-(1.7) quenches in a finite time with the only quenching node $i=1$.

Proof. Integrating (1.5) in time we find

$$
U_{i}(t)-U_{i}(0)=\int_{0}^{t} \delta^{2} U_{i}(\tau)+b_{i}\left(U_{i}^{-m}(\tau)+V_{i}^{-p}(\tau)\right) d \tau
$$

summing up the above inequality we get

$$
\sum_{i=1}^{I} h U_{i}(t)=\sum_{i=1}^{I} h U_{i}(0)+\int_{0}^{t} \frac{U_{I-1}(\tau)-U_{I}(\tau)}{h}+\frac{U_{2}(\tau)-U_{1}(\tau)}{h}-2\left(U_{1}^{-m}(\tau)+V_{1}^{-p}(\tau)\right) d \tau .
$$

From (1.5) we have

$$
\begin{array}{r}
\frac{h}{2} U_{I}(t)-\frac{h}{2} U_{I}(0)=\int_{0}^{t} \frac{U_{I-1}(\tau)-U_{I}(\tau)}{h} d \tau, \text { and } \\
\frac{h}{2} U_{1}(t)-\frac{h}{2} U_{1}(0)=\int_{0}^{t} \frac{U_{2}(\tau)-U_{1}(\tau)}{h}-\left(U_{1}^{-m}(\tau)+V_{1}^{-p}(\tau)\right) d \tau .
\end{array}
$$

Thus

$$
\frac{h}{2} U_{I}(t)+\sum_{i=2}^{I-1} h U_{i}(t)+\frac{h}{2} U_{1}(t)=\frac{h}{2} U_{I}(0)+\sum_{i=2}^{I-1} h U_{i}(0)+\frac{h}{2} U_{1}(0)-\int_{0}^{t} U_{1}^{-m}(\tau)+V_{1}^{-p}(\tau) d \tau,
$$

therefore

$$
\frac{h}{2} U_{I}(t)+\sum_{i=2}^{I-1} h U_{i}(t)+\frac{h}{2} U_{1}(t) \leq M-\left(M^{-m}+N^{-p}\right) t .
$$


Proceeding as before, we find that

$$
\frac{h}{2} V_{I}(t)+\sum_{i=2}^{I-1} h V_{i}(t)+\frac{h}{2} V_{1}(t) \leq N-\left(M^{-q}+N^{-n}\right) t,
$$

which yield a contradiction because $U_{h}$ and $V_{h}$ are positive for all times. Then there exists $0<T_{h}<\infty$ such that

$$
\lim _{t \rightarrow T^{-}} \min \left\{U_{1}(t), V_{1}(t)\right\}=0^{+}
$$

To show $i=1$ is the unique quenching node. In everything that follows $i \in\{1, \ldots, I-1\}$ and $t \in\left(0, T_{h}\right)$. Set $g\left(U_{i}(t)\right)=U_{i}^{-m}(t), f\left(V_{i}(t)\right)=V_{i}^{-p}(t), d\left(U_{i}(t)\right)=U_{i}^{-q}(t), j\left(V_{i}(t)\right)=V_{i}^{-n}(t)$, and

$$
\begin{aligned}
& Z_{i}(t)=\frac{U_{i+1}(t)-U_{i}(t)}{h}-\phi_{i}\left(g\left(U_{i}(t)\right)+f\left(V_{i}(t)\right)\right) \\
& W_{i}(t)=\frac{V_{i+1}(t)-V_{i}(t)}{h}-\phi_{i}\left(d\left(U_{i}(t)\right)+j\left(V_{i}(t)\right)\right)
\end{aligned}
$$

where $\phi_{i}, \delta^{2} \phi_{i} \geq 0, \delta^{+} \phi_{i} \leq 0, \phi_{I}=0, \phi_{1}=1, \phi_{i}\left(g\left(U_{i}(0)\right)+f\left(V_{i}(0)\right)\right) \leq \delta^{+} U_{i}(0)$ and $\phi_{i}\left(d\left(U_{i}(0)\right)+j\left(V_{i}(0)\right)\right) \leq$ $\delta^{+} V_{i}(0)$.

By means of Taylor expansions we have

$$
\begin{aligned}
\delta^{2}\left(\phi_{i} k\left(J_{i}(t)\right)\right)= & \phi_{i} k^{\prime}\left(J_{i}(t)\right) \delta^{2} J_{i}(t)+k\left(J_{i}(t)\right) \delta^{2} \phi_{i}+k^{\prime}\left(J_{i}(t)\right) \delta^{+} \phi_{i} \delta^{+} J_{i}(t)+k^{\prime}\left(J_{i}(t)\right) \delta^{-} \phi_{i} \delta^{-} J_{i}(t) \\
& +\phi_{i} \frac{\left(\delta^{+} J_{i}(t)\right)^{2}}{2} k^{\prime \prime}\left(\rho_{i}(t)\right)+\phi_{i} \frac{\left(\delta^{-} J_{i}(t)\right)^{2}}{2}\left(k^{\prime \prime}\left(\lambda_{i}(t)\right), \quad i=2, \ldots, I-1,\right. \\
\delta^{2}\left(\phi_{1} k\left(J_{1}(t)\right)\right)= & \phi_{1} k^{\prime}\left(J_{1}(t)\right) \delta^{2} J_{1}(t)+k\left(J_{1}(t)\right) \delta^{2} \phi_{1}+2 k^{\prime}\left(J_{1}(t)\right) \delta^{+} \phi_{1} \delta^{+} J_{1}(t) \\
& +\phi_{1}\left(\delta^{+} J_{1}(t)\right)^{2} k^{\prime \prime}\left(\rho_{1}(t)\right) .
\end{aligned}
$$

If we use the fact that $J_{i}, \delta^{+} J_{i}(t)$ and $\delta^{2} J_{i}(t)$ are nonnegative and the hypothesis on $\phi_{h}$, we arrive at

$$
\delta^{2}\left(\phi_{i} k\left(J_{i}(t)\right) \geq \phi_{i} k^{\prime}\left(J_{i}(t)\right) \delta^{2} J_{i}(t), i=1, \ldots, I-1\right.
$$

By using (3.3) we can get

$$
Z_{i}^{\prime}(t)-\delta^{2} Z_{i}(t) \geq \frac{b_{i}}{h}\left(g\left(U_{i}\right)+f\left(V_{i}\right)\right)+b_{i} \phi_{i} g^{\prime}\left(U_{i}\right)\left(g\left(U_{i}\right)+f\left(V_{i}\right)\right)+b_{i} \phi_{i} f^{\prime}\left(U_{i}\right)\left(d\left(U_{i}\right)+j\left(V_{i}\right)\right) .
$$

The above inequalities implies that

$$
\begin{aligned}
Z_{i}^{\prime}(t)-\delta^{2} Z_{i}(t)+ & b_{i} g^{\prime}\left(U_{i}(t)\right) Z_{i}(t)+b_{i} f^{\prime}\left(V_{i}(t)\right) W_{i}(t) \geq b_{i}\left[\frac{1}{h}\left(g\left(U_{i}(t)\right)+f\left(V_{i}(t)\right)\right)\right. \\
& +f^{\prime}\left(U_{i}(t)\right)\left(d\left(U_{i}(t)\right)+j\left(V_{i}(t)\right)\right)+g^{\prime}\left(U_{i}(t)\right)\left(g\left(U_{i}(t)\right)+f\left(V_{i}(t)\right)\right]
\end{aligned}
$$

We obtain

$$
Z_{i}^{\prime}(t)-\delta^{2} Z_{i}(t)+b_{i} g^{\prime}\left(U_{i}(t)\right) Z_{i}(t)+b_{i} f^{\prime}\left(V_{i}(t)\right) W_{i}(t) \geq 0
$$


for the parameter $h$ small enough. Thus we have

$$
\begin{aligned}
Z_{i}^{\prime}(t)-\delta^{2} Z_{i}(t)+b_{i} g^{\prime}\left(U_{i}(t)\right) Z_{i}(t)+b_{i} f^{\prime}\left(V_{i}(t)\right) W_{i}(t) & \geq 0, \\
W_{i}^{\prime}(t)-\delta^{2} W_{i}(t)+b_{i} d^{\prime}\left(U_{i}(t)\right) Z_{i}(t)+b_{i} j^{\prime}\left(V_{i}(t)\right) W_{i}(t) & \geq 0, \\
Z_{i}(0) \geq 0, W_{i}(0) & \geq 0 .
\end{aligned}
$$

Using the Lemma 2.1 we have $Z_{i}(t) \geq 0$ and $W_{i}(t) \geq 0$, for $i=1, \ldots, I-1$ and $t \in\left(0, T_{h}\right)$. This implies that $\frac{U_{i+1}(t)-U_{i}(t)}{h} \geq \phi_{i}\left(g\left(U_{i}(t)\right)+f\left(V_{i}(t)\right)\right) \geq \frac{1}{2}\left(\frac{1}{M^{m}}+\frac{1}{N^{p}}\right)$ for $i=1, \ldots, J$, with $\phi_{J}=\frac{1}{2}$, where $J \in\{2, \ldots, I-1\}$. Thus by summing we obtain

$$
U_{i}(t) \geq U_{1}+\frac{(i-1) h}{2}\left(\frac{1}{M^{m}}+\frac{1}{N^{p}}\right) \geq \frac{(i-1) h}{2}\left(\frac{1}{M^{m}}+\frac{1}{N^{p}}\right) \text { whenever } i>1 .
$$

The same happens for $V_{h}$.

Theorem 3.2. If $\lim _{t \rightarrow T_{h}^{-}} U_{1}(t)=0^{+}\left(\lim _{t \rightarrow T_{h}^{-}} V_{1}(t)=0^{+}\right)$, then $U_{h}^{\prime}(t)$ blows up $\left(V_{h}^{\prime}(t)\right.$ blows up).

Proof. Suppose $U_{h}^{\prime}(t)$ is bounded. Then, there exists a negative constant $M$ such that $U_{h}^{\prime}(t)>M$. We have

$$
\begin{aligned}
& \sum_{i=1}^{I-1} \sum_{j=1}^{i} h^{2} U_{j}^{\prime}(t)>\sum_{i=1}^{I-1} \sum_{j=1}^{i} h^{2} M \\
& \sum_{i=1}^{I-1} \sum_{j=1}^{i} h^{2} M=\sum_{i=1}^{I-1} i h^{2} M \\
&=\frac{M}{2}+\frac{h M}{2} . \\
& \sum_{i=1}^{I-1} \sum_{j=1}^{i} h^{2} U_{j}^{\prime}(t)=\sum_{i=2}^{I-1}\left(\sum_{j=2}^{i} h^{2} U_{j}^{\prime}(t)+h^{2} U_{1}^{\prime}(t)\right)+h^{2} U_{1}^{\prime}(t)
\end{aligned}
$$

From (1.5) we arrive at

$$
\sum_{i=1}^{I-1} \sum_{j=1}^{i} h^{2} U_{j}^{\prime}(t)=U_{I}(t)-U_{1}(t)-\left(V_{1}^{-p}(t)+U_{1}^{-m}(t)\right)+\frac{h}{2} U_{1}^{\prime}(t)
$$

and Lemma 2.3 we arrive at

$$
U_{I}(t)-U_{1}(t)-\left(V_{1}(t)^{-p}+U_{1}(t)^{-m}\right)>\frac{M}{2}+h M
$$

As $t \rightarrow T_{h}^{-}$, the left-hand side tends to infinity while the right-side is finite. This contradiction shows that $U_{h}^{\prime}$ blows up. 


\section{Simultaneous Vs. NON-SimultaneOUS QUENChing}

In this Section we consider $\left(U_{h}, V_{h}\right)$ the solution of (1.5)-(1.7) with $h$ fixed, and we give some sufficient conditions for the existence of simultaneous and non-simultaneous quenching.

Theorem 4.1. If $U_{h}$ quenches and $V_{h}$ does not quench in (1.5)-(1.7) then $q<m+1$.

Proof. As $V_{h}$ does not quench, by (1.5) there exists $c>0$ such that

$$
U_{1}^{\prime}(t) \geq-c U_{1}^{-m}(t)
$$

integrating this inequality from $t$ to $T_{h}$, we get

$$
U_{1}(t) \leq C\left(T_{h}-t\right)^{\frac{1}{m+1}}, \text { where } C=((m+1) c)^{1 /(m+1)}
$$

By using (4.1) and (1.6), we obtain

$$
V_{1}^{\prime}(t) \leq \delta^{2} V_{1}(t)-b_{1}\left(V_{1}^{-n}(t)+C\left(T_{h}-t\right)^{-\frac{q}{m+1}}\right) .
$$

Thus $V_{1}\left(T_{h}\right) \leq C_{1}-C \int_{0}^{T_{h}}\left(T_{h}-t\right)^{-\frac{q}{m+1}} d t$. We can see that this integral diverges if $q \geq m+1$, which is a contradiction and the proof is completed.

Corollary 4.1. Simultaneous quenching happens if $q \geq m+1, p \geq n+1$.

Lemma 4.1. Let $\left(U_{h}, V_{h}\right)$ be the solution of (1.5)-(1.7). Assume that $U_{h}$ quenches at time $T_{h}$ ( $V_{h}$ quenches at time $T_{h}$ ) and

$$
\begin{gathered}
\delta^{2} \varphi_{1, i}-b_{i}\left(\varphi_{1, i}^{-m}+\varphi_{2, i}^{-p}\right)+c\left(\varphi_{1, i}^{-m}+\varphi_{2, i}^{-p}\right) \leq 0, \\
\delta^{2} \varphi_{2, i}-b_{i}\left(\varphi_{1, i}^{-q}+\varphi_{2, i}^{-n}\right)+c\left(\varphi_{1, i}^{-q}+\varphi_{2, i}^{-n}\right) \leq 0 .
\end{gathered}
$$

Then there exists a positive constant $C$ such that for $t \in\left(0, T_{h}\right)$

$$
\begin{gathered}
\frac{U_{1}(t)^{m+1}}{C(m+1)} \geq T_{h}-t \quad\left(\frac{V_{1}(t)^{n+1}}{C(n+1)} \geq T_{h}-t\right), \\
U_{1}(t) \geq C\left(T_{h}-t\right)^{\frac{1}{m+1}} \quad\left(V_{1}(t) \geq C\left(T_{h}-t\right)^{\frac{1}{n+1}}\right) .
\end{gathered}
$$

Proof. Set for $i=1, \ldots, I, t \in\left(0, T_{h}\right)$,

$$
Z_{i}(t)=U_{i}^{\prime}(t)+c\left(U_{i}^{-m}(t)+V_{i}^{-p}(t)\right) \text { and } W_{i}(t)=V_{i}^{\prime}(t)+c\left(U_{i}^{-q}(t)+V_{i}^{-n}(t)\right)
$$

A straightforward calculation gives

$$
\begin{array}{r}
Z_{i}^{\prime}(t)-\delta^{2} Z_{i}(t)+\alpha_{i}(t) Z_{i}(t)+\beta_{i}(t) W_{i}(t) \leq 0, \quad i=1, \ldots, I, t \in\left(0, T_{h}\right), \\
W_{i}^{\prime}(t)-\delta^{2} W_{i}(t)+a_{i}(t) Z_{i}(t)+b_{i}(t) W_{i}(t) \leq 0, \quad i=1, \ldots, I, t \in\left(0, T_{h}\right), \\
Z_{i}(0) \leq 0, \quad W_{i}(0) \leq 0, \quad i=1, \ldots, I .
\end{array}
$$


By virtue of Lemma 2.1

$$
Z_{i}(t) \leq 0, W_{i}(t) \leq 0, \quad i=1, \ldots, I, t \in\left(0, T_{h}\right)
$$

Thus we get

$$
U_{i}^{\prime}(t) \leq-c U_{i}^{-m}(t) \text { and } V_{i}^{\prime}(t) \leq-c V_{i}^{-n}(t), \quad i=1, \ldots, I, t \in\left(0, T_{h}\right)
$$

Assume that $U_{h}$ quenches ( $V_{h}$ quenches), integrating (4.5) from $t$ to $T_{h}$, we arrive at

$$
\frac{U_{1}(t)^{m+1}}{C(m+1)} \geq T_{h}-t \quad\left(\frac{V_{1}(t)^{n+1}}{C(n+1)} \geq T_{h}-t\right)
$$

which implies

$$
U_{1}(t) \geq C\left(T_{h}-t\right)^{\frac{1}{m+1}} \quad\left(V_{1}(t) \geq C\left(T_{h}-t\right)^{\frac{1}{n+1}}\right) .
$$

Theorem 4.2. If $p<n+1$, then there exist initial data such that $V_{h}$ quenches but $U_{h}$ doesn't.

Proof. We argue by contradiction. Assuming that $U_{h}$ and $V_{h}$ quench simultaneously at time $T_{h}$ for any initial data. We have

$$
\int_{0}^{t} U_{1}^{\prime}(s) d s \geq \int_{0}^{T_{h}} U_{1}^{\prime}(s) d s=\frac{2}{h^{2}} \int_{0}^{T_{h}} U_{2}(s)-U_{1}(s) d s-\frac{2}{h} \int_{0}^{T_{h}} U_{1}^{-m}(s)+V_{1}^{-p}(s) d s
$$

By using the Lemma 4.1, we obtain

$$
U_{1}(t) \geq U_{1}(0)+\frac{2}{h^{2}} \int_{0}^{T_{h}} U_{2}(s)-U_{1}(s) d s-\frac{2 C}{h} \int_{0}^{T_{h}}\left(T_{h}-s\right)^{-\frac{m}{m+1}}+\left(T_{h}-s\right)^{-\frac{p}{n+1}} d s
$$

As $p<n+1$ this integral is converged and

$$
U_{1}(t) \geq C_{1}-C_{2} T_{h}^{\frac{1}{m+1}}-C_{3} T_{h}^{\frac{n+1-p}{n+1}}, \quad \text { with } C_{1}, C_{2}, C_{3}>0 .
$$

By summation of (1.6) we observe that

$$
\begin{aligned}
-\frac{h}{2} V_{1}^{\prime}(t)-\frac{h}{2} V_{I}^{\prime}(t)-\sum_{i=2}^{I-1} h V_{i}^{\prime}(t) & =U_{1}^{-q}(t)+V_{1}^{-n}(t), \\
-\frac{h}{2} V_{1}^{\prime}(t)-\frac{h}{2} V_{I}^{\prime}(t)-\sum_{i=2}^{I-1} h V_{i}^{\prime}(t) & \geq U_{1}^{-q}(0)+V_{1}^{-n}(0)
\end{aligned}
$$

integrate (4.6) from 0 to $T_{h}$, we can obtain

$$
V_{I}(0)\left(U_{1}^{-q}(0)+V_{1}^{-n}(0)\right)^{-1} \geq T_{h},
$$

then if $T_{h}$ is small enough (depending on $U_{h}(0)$ and $\left.V_{h}(0)\right), U_{1}\left(T_{h}\right) \geq c_{0}>0$. We have a contradiction with the hypothesis that $U_{h}$ quenches and the result is obtained as desired. 
Theorem 4.3. If $q \leq \frac{n(m+1)}{n+1}$ and $p \geq n+1\left(p \leq \frac{m(n+1)}{m+1}\right.$ and $\left.q \geq m+1\right)$ then $U_{h}\left(V_{h}\right)$ quenches alone under any positive initial data.

Proof. Assume that there exists initial data such that $U_{h}$ and $V_{h}$ quench simultaneously at time $T_{h}$. Without lost of generality, we can suppose that this initial data satisfies (4.2)-(4.3). According to (1.6)

$$
\begin{array}{r}
V_{1}^{\prime}(t)=\delta^{2} V_{1}(t)-b_{1}\left(U_{1}^{-q}(t)+V_{1}^{-n}(t)\right), \\
V_{1}^{\prime}(t) \geq-b_{1}\left(U_{1}^{-q}(t)+V_{1}^{-n}(t)\right), \\
V_{1}(t) \leq b_{1} \int_{t}^{T_{h}} U_{1}^{-q}(s)+V_{1}^{-n}(s) d s .
\end{array}
$$

From Lemma 4.1 we know $U_{1}(t) \geq C\left(T_{h}-t\right)^{\frac{1}{m+1}}, V_{1}(t) \geq C\left(T_{h}-t\right)^{\frac{1}{n+1}}$, moreover $q \leq \frac{n(m+1)}{n+1}$. Hence there exists $C^{\prime}>0$ such that $V_{1}(t) \leq C^{\prime}\left(T_{h}-t\right)^{\frac{1}{n+1}}$. Let us consider (1.5)

$$
\begin{aligned}
U_{1}^{\prime}(t) & =\delta^{2} U_{1}(t)-b_{1}\left(U_{1}^{-m}(t)+V_{1}^{-p}(t)\right), \\
U_{1}^{\prime}(t) & \leq \delta^{2} U_{1}(t)-b_{1} V_{1}^{-p}(t), \\
U_{1}^{\prime}(t) & \leq \delta^{2} U_{1}(t)-b_{1} C^{\prime-p}\left(T_{h}-t\right)^{-\frac{p}{n+1}} .
\end{aligned}
$$

Integrating both sides from 0 to $T_{h}$, we obtain

$$
-U_{1}(0) \leq c_{1}-c_{2} \int_{0}^{T_{h}}\left(T_{h}-t\right)^{-\frac{p}{n+1}} d t
$$

We can see that the integral diverges if $p \geq n+1$, which is a contradiction. The result is obtained.

Remark 4.1. Let $\left(U_{h}, V_{h}\right)$ be the solution of (1.5)-(1.7) such that the initial data satisfies (4.2)-(4.3). We can see of the Lemma 4.1 and the proof of Theorem 4.3 that if $U_{h}\left(V_{h}\right)$ quenches at time $T_{h}$, then $U_{1}(t) \sim\left(T_{h}-t\right)^{\frac{1}{m+1}}\left(V_{1}(t) \sim\left(T_{h}-t\right)^{\frac{1}{n+1}}\right)$ for $t$ close enough to $T_{h}$.

\section{Convergence of the Semidiscrete quenching time}

In this section, we study the convergence of the semidiscrete quenching time. Now we will show that for each fixed time interval $[0, T]$ where $(u, v)$ is defined, the solution $\left(U_{h}, V_{h}\right)$ of (1.5)-(1.7) approximates $(u, v)$ when the mesh parameter $h$ goes to zero. We denote

$$
\begin{gathered}
u_{h}(t)=\left(u\left(x_{1}, t\right), \ldots, u\left(x_{I}, t\right)\right)^{T}, v_{h}(t)=\left(v\left(x_{1}, t\right), \ldots, v\left(x_{I}, t\right)\right)^{T}, \\
\left\|U_{h}(t)\right\|_{\infty}=\max _{1 \leq i \leq I}\left|U_{i}(t)\right|, \quad\left\|U_{h}(t)\right\|_{\text {inf }}=\min _{1 \leq i \leq I}\left|U_{i}(t)\right| .
\end{gathered}
$$

Theorem 5.1. Assume that the problem (1.1)-(1.4) has solution

$(u, v) \in\left(C^{4,1}\left([0,1] \times\left[0, T^{*}\right]\right)\right)^{2}$ and the initial data $\left(\varphi_{1, h}, \varphi_{2, h}\right)$ at (1.5)-(1.7) verifies

$$
\left\|\varphi_{1, h}-u_{h}(0)\right\|_{\infty}=o(1),\left\|\varphi_{2, h}-v_{h}(0)\right\|_{\infty}=o(1) \quad h \rightarrow 0 .
$$


Then, for $h$ small enough, the semidiscrete problem (1.5)-(1.7) has a unique solution $\left(U_{h}, V_{h}\right) \in$ $\left(C^{1}\left(\left[0, T^{*}\right], \mathbf{R}^{I}\right)\right)^{2}$ such that

$$
\begin{aligned}
& \max _{t \in\left[0, T^{*}\right]}\left\|U_{h}(t)-u_{h}(t)\right\|_{\infty}=O\left(\left\|\varphi_{1, h}-u_{h}(0)\right\|_{\infty}+\left\|\varphi_{2, h}-v_{h}(0)\right\|_{\infty}+h^{2}\right) \text {, as } h \rightarrow 0, \\
& \max _{t \in\left[0, T^{*}\right]}\left\|V_{h}(t)-v_{h}(t)\right\|_{\infty}=O\left(\left\|\varphi_{1, h}-u_{h}(0)\right\|_{\infty}+\left\|\varphi_{2, h}-v_{h}(0)\right\|_{\infty}+h^{2}\right) \text {, as } h \rightarrow 0 .
\end{aligned}
$$

Proof. Let $\sigma>0$ be such that

$$
\left(\|u\|_{\infty},\|v\|_{\infty}\right)<\sigma, t \in\left[0, T^{*}\right] .
$$

Then the problem (1.5)-(1.7) has for each $h$, a unique solution $\left(U_{h}, V_{h}\right) \in\left(C^{1}\left(\left[0, T^{*}\right], \mathbf{R}^{I}\right)\right)^{2}$. Let $t(h) \leq T^{*}$ be the greatest value of $t>0$ such that

$$
\max \left\{\left\|U_{h}(t)-u_{h}(t)\right\|_{\infty},\left\|V_{h}(t)-v_{h}(t)\right\|_{\infty}\right\}<1
$$

The relation (5.1) implies $t(h)>0$ for $h$ small enough. Using the triangle inequality, we obtain

$$
\left\|U_{h}(t)\right\|_{\infty} \leq 1+\sigma \text { and, }\left\|V_{h}(t)\right\|_{\infty} \leq 1+\sigma \text { for } t \in(0, t(h)) .
$$

Let $\left(e_{1, h}, e_{2, h}\right)(t)=\left(U_{h}-u_{h}, V_{h}-v_{h}\right)(t), \forall t \in\left[0, T^{*}\right]$ be the discretization error. these error functions verify

$$
\begin{gathered}
e_{1, i}^{\prime}(t)=\delta^{2} e_{1, i}(t)+m b_{i}\left(\theta_{i}(t)\right)^{-m-1} e_{1, i}(t)+p b_{i}\left(\Theta_{i}(t)\right)^{-p-1} e_{2, i}(t)+O\left(h^{2}\right), \\
e_{2, i}^{\prime}(t)=\delta^{2} e_{2, i}(t)+q b_{i}\left(\theta_{i}(t)\right)^{-q-1} e_{1, i}(t)+n b_{i}\left(\Theta_{i}(t)\right)^{-n-1} e_{2, i}(t)+O\left(h^{2}\right),
\end{gathered}
$$

where $\theta_{i}(t)$ and $\Theta_{i}(t)$ lie, respectively, between $U_{i}(t)$ and $u\left(x_{i}, t\right)$, and between $V_{i}(t)$ and $v\left(x_{i}, t\right)$, for $i \in$ $\{1, \ldots, I\}$. Using (5.2) and (5.4), there exist $\mathrm{K}$ and $\mathrm{L}$ positive constants such that

$$
\begin{gathered}
e_{1, i}^{\prime}(t) \leq \delta^{2} e_{1, i}(t)+b_{i} L\left|e_{1, i}(t)\right|+b_{i} L\left|e_{2, i}(t)\right|+K h^{2}, \\
e_{2, i}^{\prime}(t) \leq \delta^{2} e_{2, i}(t)+b_{i} L\left|e_{1, i}(t)\right|+b_{i} L\left|e_{2, i}(t)\right|+K h^{2}
\end{gathered}
$$

let $(z, w) \in\left(C^{4,1}\left([0,1],\left[0, T^{*}\right]\right)\right)^{2}$ be such that

$z(x, t)=\left(\left\|\varphi_{1, h}-u_{h}(0)\right\|_{\infty}+\left\|\varphi_{2, h}-v_{h}(0)\right\|_{\infty}+Q h^{2}\right) e^{(M+2) t-(1-x)^{2}}$ and $w=z, \forall(x, t) \in[0,1] \times\left[0, T^{*}\right]$, with $M, Q$ positive constants. We can prove by the Lemma 2.2 that

$$
\left|e_{1, i}(t)\right|<z\left(x_{i}, t\right), \quad\left|e_{2, i}(t)\right|<w\left(x_{i}, t\right), 1 \leq i \leq I, \text { for } t \in(0, t(h)) .
$$

We deduce that

$$
\begin{aligned}
\left\|U_{h}(t)-u_{h}(t)\right\|_{\infty} & \leq\left(\left\|\varphi_{1, h}-u_{h}(0)\right\|_{\infty}+\left\|\varphi_{2, h}-v_{h}(0)\right\|_{\infty}+Q h^{2}\right) e^{(M+2) t}, \\
\left\|V_{h}(t)-v_{h}(t)\right\|_{\infty} & \leq\left(\left\|\varphi_{1, h}-u_{h}(0)\right\|_{\infty}+\left\|\varphi_{2, h}-v_{h}(0)\right\|_{\infty}+Q h^{2}\right) e^{(M+2) t},
\end{aligned}
$$

for $t \in(0, t(h))$. Suppose that $T^{*}>t(h)$ from (5.3), we obtain

$$
1=\left\|U_{h}(t(h))-u_{h}(t(h))\right\|_{\infty} \leq\left(\left\|\varphi_{1, h}-u_{h}(0)\right\|_{\infty}+\left\|\varphi_{2, h}-v_{h}(0)\right\|_{\infty}+Q h^{2}\right) e^{(M+2) t}
$$


Since the term on the right hand side of the above inequality goes to zero as $\mathrm{h}$ tends to zero, we deduce that, $1 \leq 0$, which is impossible. Hence we have $t(h)=T^{*}$, and the proof is completed.

Theorem 5.2. Let $(u, v) \in\left(C^{4,1}([0,1] \times[0, T))\right)^{2}$ be solution of (1.1)-(1.4) with quenches time $T$ and the initial data at (1.5)-(1.7) satisfies (4.2)-(4.3) and (5.1). Then the solution $\left(U_{h}, V_{h}\right)$ of (1.5)-(1.7) quenches in a finite time $T_{h}$ and we have

$$
\lim _{h \rightarrow 0} T_{h}=T
$$

Proof. From Theorem 3.1, $\left(U_{h}, V_{h}\right)$ quenches in a finite time $T_{h}$. Assume that $U_{h}$ quenches.

Set $\varepsilon>0$. There exists $\eta>0$ such that

$$
\frac{y^{1+m}}{C(m+1)} \leq \frac{\varepsilon}{2}, \quad 0 \leq y \leq \eta
$$

There exists a time $T_{0} \in\left(T-\frac{\varepsilon}{2}, T\right)$ such that $0<\left|u\left(x_{i}, t\right)\right| \leq \frac{\eta}{2}$, for $i=1, \ldots, I, t \in\left[T_{0}, T\right)$. Setting $T_{1}=\frac{T_{0}+T}{2}$, it is not hard to see that $0<\left\|u\left(x_{i}, t\right)\right\|_{\text {inf }}$, for $t \in\left[0, T_{1}\right]$. From Theorem 5.1 , it follows that for $h$ sufficiently small

$$
\left\|U_{h}(t)-u_{h}(t)\right\|_{\infty} \leq \frac{\eta}{2}
$$

Applying the triangle inequality, we get

$$
\left\|U_{h}\left(T_{1}\right)\right\|_{\text {inf }} \leq\left\|U_{h}\left(T_{1}\right)-u_{h}\left(T_{1}\right)\right\|_{\infty}+\left\|u_{h}\left(T_{1}\right)\right\|_{\text {inf }} \leq \eta
$$

Since $U_{h}$ quenches, we can deduce from Lemma 4.1 and (5.5) that

$$
\left|T_{h}-T\right| \leq\left|T_{h}-T_{1}\right|+\left|T_{1}-T\right| \leq \frac{\left\|U_{h}\left(T_{1}\right)\right\|_{\text {inf }}^{1+m}}{C(m+1)}+\frac{\varepsilon}{2} \leq \varepsilon .
$$

The case where $V_{h}$ quenches is analogous.

\section{Numerical Experiments}

In this section, we present some numerical approximations to the quenching time of (1.5)-(1.7) for the initial data $\varphi_{1, i}=\varphi_{2, i}=1+\frac{4}{\Pi} \sin \left(\frac{\Pi}{2}(i-1) h\right)$ for $i=1, \ldots, I-1$, with different values of $m, n, p$ and $q$. We also consider the implicit scheme below

$$
\begin{aligned}
& \frac{U_{i}^{(k+1)}-U_{i}^{(k)}}{\Delta t_{h}^{k}}=\delta^{2} U_{i}^{(k+1)}-b_{i}\left(\left(U_{i}^{(k)}\right)^{-m}+\left(V_{i}^{(k)}\right)^{-p}\right), \quad 1 \leq i \leq I, \\
& \frac{V_{i}^{(k+1)}-V_{i}^{(k)}}{\Delta t_{h}^{k}}=\delta^{2} V_{i}^{(k+1)}-b_{i}\left(\left(U_{i}^{(k)}\right)^{-q}+\left(V_{i}^{(k)}\right)^{-n}\right), \quad 1 \leq i \leq I, \\
& U_{i}^{(0)}=\varphi_{1, i}, \quad V_{i}^{(0)}=\varphi_{2, i} \quad 1 \leq i \leq I,
\end{aligned}
$$

where $k \geq 0, \Delta t_{h}^{k}=h^{2} \min \left\{\left\|U_{h}^{(k)}\right\|_{\text {inf }}^{m+1},\left\|U_{h}^{(k)}\right\|_{\text {inf }}^{q+1},\left\|V_{h}^{(k)}\right\|_{\text {inf }}^{p+1},\left\|V_{h}^{(k)}\right\|_{\text {inf }}^{n+1}\right\}$. 
Definition 6.1. We say that the discrete solution $\left(U_{h}^{(k)}, V_{h}^{(k)}\right)$ of the implicit scheme quenches in a finite time if

$\lim _{k \rightarrow \infty} \inf \left\{\left\|U_{h}^{(k)}\right\|_{\text {inf }},\left\|V_{h}^{(k)}\right\|_{\text {inf }}\right\}=0$ and the series $\sum_{k=0}^{+\infty} \Delta t_{h}^{k}$ converges. The quantity $t_{h}^{k}=\sum_{j=0}^{k-1} \Delta t_{h}^{j}$ is called the numerical quenching time of the solution $\left(U_{h}^{(k)}, V_{h}^{(k)}\right)$ and $T_{h}=\sum_{k=0}^{+\infty} \Delta t_{h}^{k}$ is called the numerical quenching time of the solution $\left(U_{h}, V_{h}\right)$.

In Tables 1, 2 and 3, in rows, we present the numerical quenching times, the numbers of iterations and the orders of the approximations corresponding to meshes of $16,32,64,128,256,512$, 1024. We take for the numerical quenching time $T_{h}=\sum_{k=0}^{+\infty} \Delta t_{h}^{k}$ which is computed at the first time when $\Delta t_{h}^{k}=\left|t_{h}^{k+1}-t_{h}^{k}\right| \leq$ $10^{-16}$. The order(s) of the method is computed from

$$
s=\frac{\log \left(\left(T_{4 h}-T_{2 h}\right) /\left(T_{2 h}-T_{h}\right)\right)}{\log (2)}, \quad \text { where } h=1 /(I-1) .
$$

TABLE 1. Numerical quenching times obtained with the implicit

Euler method for $m=0.5, p=1$, $q=2, n=0.5$.

\begin{tabular}{|c|c|c|c|}
\hline$I$ & $T_{h}$ & $k$ & $s$ \\
\hline 16 & 0.15390794 & 34896 & - \\
\hline 32 & 0.14878519 & 48454 & - \\
\hline 64 & 0.14737661 & 66676 & 1.86 \\
\hline 128 & 0.14699264 & 92814 & 1.87 \\
\hline 256 & 0.14688843 & 137205 & 1.88 \\
\hline 512 & 0.14686025 & 238258 & 1.89 \\
\hline 1024 & 0.14685267 & 545941 & 1.89 \\
\hline
\end{tabular}

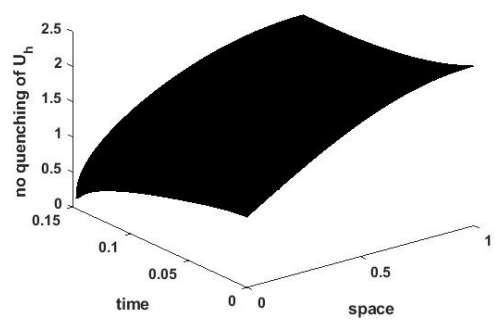

TABLE 2. Numerical quenching times obtained with the explicit Euler method for $m=1, p=2.5$, $q=0.5, n=1$.

\begin{tabular}{|c|c|c|c|}
\hline$I$ & $T_{h}$ & $k$ & $s$ \\
\hline 16 & 0.13630655 & 168 & - \\
\hline 32 & 0.13147195 & 484 & - \\
\hline 64 & 0.13016571 & 1540 & 1.89 \\
\hline 128 & 0.12981187 & 5384 & 1.88 \\
\hline 256 & 0.12971578 & 20063 & 1.88 \\
\hline 512 & 0.12968969 & 77515 & 1.88 \\
\hline 1024 & 0.12968263 & 305050 & 1.88 \\
\hline
\end{tabular}

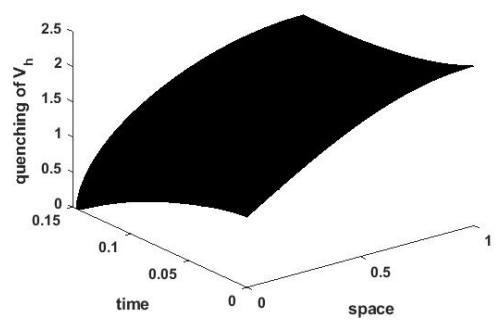

Figure 1. On the left, no quenching of $U_{h}$ and on the right, quenching of $V_{h}$ for $m=0.5$, $p=1, q=2, n=0.5$. 
TABLE 3. Numerical quenching times obtained with the implicit Euler method for $m=0.3, p=2, q=2$, $n=0.3$.

\begin{tabular}{|c|c|c|c|}
\hline$I$ & $T_{h}$ & $k$ & $s$ \\
\hline 16 & 0.12862938 & 127 & - \\
\hline 32 & 0.12271047 & 372 & - \\
\hline 64 & 0.12106075 & 1213 & 1.84 \\
\hline 128 & 0.12060846 & 4323 & 1.87 \\
\hline 256 & 0.12048544 & 16309 & 1.88 \\
\hline 512 & 0.12045217 & 63432 & 1.89 \\
\hline 1024 & 0.12044321 & 250457 & 1.89 \\
\hline
\end{tabular}
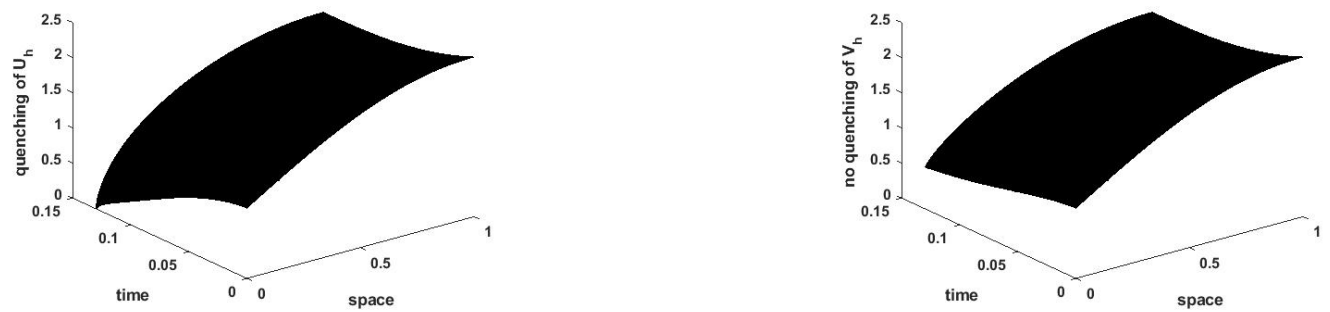

Figure 2. On the left, quenching of $U_{h}$ and on the right, no quenching of $V_{h}$ for $m=1$, $p=2.5, q=0.5, n=1$.
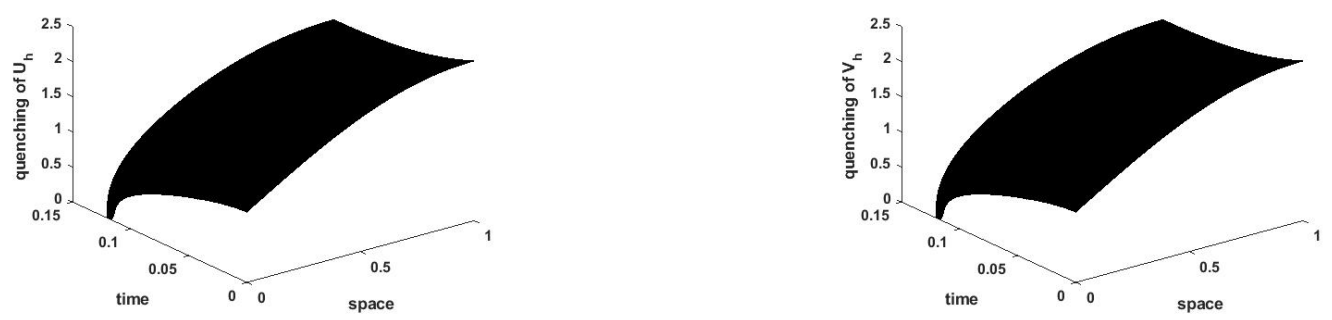

FigurE 3. On the left, quenching of $U_{h}$ and on the right, quenching of $V_{h}$ for $m=0.3$, $p=2, q=2, n=0.3$.

Remark 6.1. The various tables of our numerical results show that there is a relationship between the quenching time and flows on the boundaries. If we consider the problem (1.5)-(1.7) in the case where the initial data $\varphi_{2, i}=\varphi_{1, i}=1+\frac{4}{\Pi} \sin \left(\frac{\Pi}{2}(i-1) h\right), i=1, \ldots, I$, from figures 1-3, we observe respectively the quenching of $\left(U_{h}, V_{h}\right)$. From tables 1-3, we observe the convergence of quenching time $T_{h}$ of the solution of (1.5)-(1.7), since the rate of convergence is near 2. This result does not surprise us because of the result 
established in the previous section. Moreover we can see that of the figure $1, V_{h}$ quenches while $U_{h}$ doesn't when $p<n+1$, of the figure $2, U_{h}$ quenches while $V_{h}$ doesn't when $q \leq \frac{n(m+1)}{n+1}$ and $p \geq n+1$ and of the figure $3, U_{h}$ and $V_{h}$ quench simultaneously when $p \geq n+1$ and $q \geq m+1$. These numerical results are in fact consistent with the Corollary 4.1, Theorem 4.2 and Theorem 4.3.

\section{Conclusion}

In this work, we proposed a semi-discrete scheme, based on finite difference method in space for system of heat equations, coupled by nonlinear boundary flux. The stability and the convergence of semi-discrete scheme are proved respectively. Under some conditions the semidiscrete scheme reproduces well the conditions for the quenching, quenching set and simultaneous and non-simultaneous quenching. The analysis in this paper can be extended to more general to some systems of nonlinear parabolic equations.

\section{REFERENCES}

[1] K.A. Adou, K.A. Touré, A. Coulibaly, Numerical study of the blow-up time of positive solutions of semilinear heat equations, Far East J. Appl. Math., 4 (2018), 91-308.

[2] T. K. Boni, Extinction for discretizations of some semilinear parabolic equations, C. R. Acad. Sci. Paris, 333 (2001), 79-800.

[3] T. K. Boni, H. Nachid, D. Nabongo, Quenching time of semilinear heat equations, Miskolc Math. Notes, 11 (2010), $27-41$.

[4] C.Y. Chan, S.I. Yuen, Parabolic problems with nonlinear absorptions and releases at the boundaries, Appl. Math. comput., 121 (2001), 203-209.

[5] K.B. Edja, K.A. Touré, B. J.-C. Koua, Numerical Blow-up for a Heat Equation with Nonlinear Boundary Conditions, J. Math. Res., 10 (2018), 119-128.

[6] R. Ferreira, A. de Pablo, M.P. LLanos, J.D. Rossi, Incomplete quenching in a system of heat equations coupled at the boundary, J. Math. Anal. Appl., 346 (2008), 1145-154.

[7] R.H. Ji, C.Y. Quc, L.D. Wang, Simultaneous and non-simultaneous quenching for coupled parabolic system, Appl. Anal., 94 (2015), 233-250.

[8] H. Kawarada, On Solutions of Initial-Boundary Problem for $u_{t}=u_{x x}+\frac{1}{1-u}$, Publ. RIMS, Kyoto Univ., 10 (1975), $729-736$.

[9] H.A. Levine, The Quenching of Solutions of Linear Parabolic and Hyperbolic Equations with Nonlinear Boundary Conditions, SIAM J. Math. Anal., 4 (1983), 1139-1153.

[10] H.A. Levine, J.T. Montgomer The quenching of solutions of some nonlinear parabolic equations, SIAM J. Math. Anal., 11 (1980), 842-847.

[11] Liang K.W, Lin AP, Tan RCE. Numerical Solution of Quenching Problems Using Mesh-Dependent Variable Temporal Steps, Appl. Numer. Math., 57 (2007), 791-800.

[12] D. Nabongo, T.K. Boni, Quenching for semidiscretizations of a heat equation with a singular boundary condition, Asymptotic Anal., 59 (2008), 27-38.

[13] K.C. N'dri, K.A. Touré, G. Yoro, Numerical blow-up time for a parabolic equation with nonlinear boundary conditions, Int. J. Numer. Methods Appl., 17 (2018), 141-160.

[14] H. Pei, Z. Li, Quenching for a parabolic system with general singular terms, J. Nonlinear Sci. Appl., 7 (2016), 1-10.

[15] B. Seluk, Quenching behavior of a semilinear reaction-diffusion system with singular boundary condition, Turk. J. Math., 40 (2016), 166-180. 
[16] M. Taha, K. Toure, E. Mensah, Numerical approximation of the blow-up time for a semilinear parabolic equation with nonlinear boundary equation, Far East J. Appl. Math., 60 (2012), 125-167.

[17] S.N. Zheng, X.F. Song, Quenching rates for heat equations with coupled nonlinear boundary flux, Sci. China Ser. A., 51 (2008), 1631-1643. 